\title{
In(III)-TMSBr-Catalyzed Cascade Reaction of Diarylalkynes with Acrylates for the Synthesis of Aryldihydronaphthalene Derivatives
}

\author{
Qiu-Chi Zhang ${ }^{1,+}$, Wen-Wei Zhang ${ }^{2,+}$, Liang Shen ${ }^{1}$, Zhi-Liang Shen ${ }^{3}$ and Teck-Peng Loh ${ }^{1,3, *}$ \\ 1 Division of Chemistry and Biological Chemistry, School of Physical and Mathematical Sciences, \\ Nanyang Technological University, Singapore 637371, Singapore; zhan0413@e.ntu.edu.sg (Q.-C.Z.); \\ lshen003@e.ntu.edu.sg (L.S.) \\ 2 BGI, BGI-Shenzhen, Shenzhen 518083, China; zhangww@genomics.cn \\ 3 Institute of Advanced Synthesis, School of Chemistry and Molecular Engineering, Jiangsu National \\ Synergetic Innovation Center for Advanced Materials, Nanjing Tech University, Nanjing 210009, China; \\ ias_zlshen@njtech.edu.cn \\ * Correspondence: teckpeng@ntu.edu.sg; Tel.: +65-6316-8899 \\ + These authors contributed equally to this work.
}

Academic Editors: Akio Baba and Makoto Yasuda

Received: 20 March 2018; Accepted: 20 April 2018; Published: 23 April 2018

\begin{abstract}
A combined Lewis acid system comprising of two or more Lewis acids occasionally exhibits augmented catalytic activity in organic transformations which are otherwise unrealizable by either of the components exclusively. On the other hand, the efficient construction of multiple new C-C bonds and polycyclic structures in minimal steps remains a subject of great interest in both academia and industry. Herein we report an efficient method to assemble aryldihydronaphthalene derivatives via a cascade reaction of diarylalkynes with acrylates under the catalysis of a combined Lewis acid derived from $\mathrm{In}(\mathrm{III})$ salt and TMSBr.
\end{abstract}

Keywords: indium; combined Lewis acid; catalysis; cascade reaction; aryldihydronaphthalene

\section{Introduction}

In the context of green chemistry, efficient methods to forge multiple new $\mathrm{C}-\mathrm{C}$ bonds and to introduce molecular complexity in minimal steps have been a long-standing and important goal in the development of modern organic chemistry [1,2]. In this regard, aryldihydronaphthalene derivatives are particularly attractive targets mainly due to their widespread occurrence in natural products and bioactive compounds (Figure 1) [3-7]. They are also employed as fluorescent ligands in biochemistry studies or building blocks towards the synthesis of several biologically-active cyclic molecules [8-11]. Consequently, their significant roles have driven the establishment of several synthetic methodologies for their preparation. Prominently, the intramolecular hydroarylation of 4-phenyl-1-butyne or its derivatives is one of the most versatile protocols for the construction of aryldihydronaphthalene derivatives [12-15]. Since the pioneering studies by Fujiwara et al. [16,17], numerous catalytic methods have been developed in this field in which a series of transition metals, Lewis and Bronsted acids have been found effective for catalyzing the hydroarylation [18-39]. More recently, Corey [24] and Pérez Sestelo and Martínez [38,39] have independently reported the formation of six-membered oxa- and carbocycles by an In(III)-catalyzed hydroarylation of acetylenic substrates. 
<smiles>COc1ccc2c(c1)CCC(c1ccccc1)=C2c1ccc(OCCC2CCCC2)cc1</smiles><smiles>COc1cc2c(cc1O)[C@@](CO)(c1cccc(O)c1OC)C(CO)(CO)C=C2CO</smiles>

Negundin B<smiles>COc1ccc2c(c1O)CCC=C2c1cc(OC)c(OC)c(OC)c1</smiles>

Oxi 6196<smiles>O=C1OCC2Cc3ccc4c(c3C(c3ccc5c(c3)OCO5)=C12)OCO4</smiles>

7,8-dihydrotaiwanin C<smiles>O=C1OCC2Cc3cc4c(cc3C(c3ccc5c(c3)OCO5)=C12)OCO4</smiles>

7,8-dihydroretrohelioxanthin<smiles>COc1cc2c(cc1OC)C(c1ccc3c(c1)OCO3)=C1C(=O)OCC1C2</smiles>

Collinusin

Figure 1. Representative natural products and bioactive compounds containing aryldihydronaphthalene structures [3-7].

On the other hand, although indium(III) salts $\left(\operatorname{InX}_{3}\left(\mathrm{X}=\mathrm{Cl}, \mathrm{Br}, \mathrm{I}, \mathrm{OTf}, \mathrm{NTf}_{2}\right.\right.$, etc.)) have been widely applied as Lewis acids in organic synthesis [40-42], their relatively weak Lewis acidity nonetheless limit their applications. Unlike other group III elements, such as aluminum and boron, trimethylsilyl halide is often employed together with $\operatorname{InX}_{3}$ as a robust combined Lewis acid catalyst, as observed in various reactions developed by Baba [43,44], Lee [45], Corey [24,46], our group [47-49], and others. As a continuation of our research interest in the application of indium in organic synthesis [47-51], herein we wish to report the first example of the In(III)-TMSBr-catalysed cascade reaction of diarylalkynes with acrylates to access a series of dihydronaphthalene derivatives in a one-pot manner (Scheme 1).
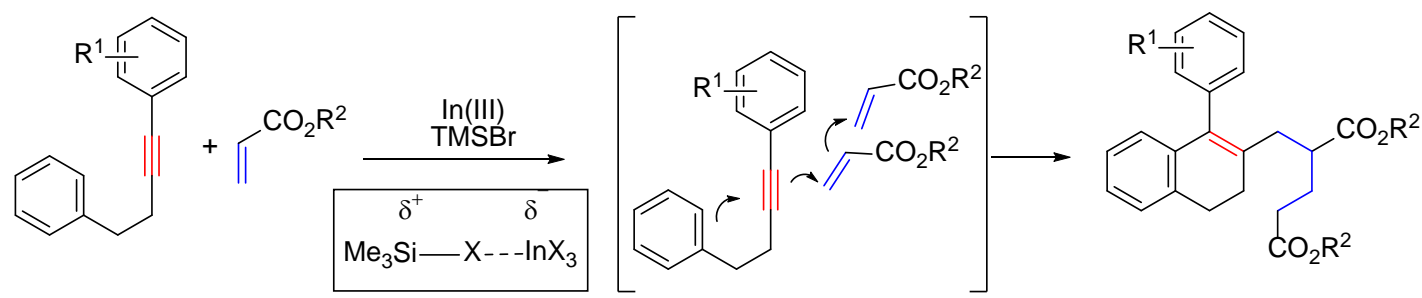

combined Lewis acid

Scheme 1. In(III)-TMSBr-catalysed cascade reaction of diarylalkynes with acrylates for the synthesis of dihydronaphthalene derivatives.

\section{Results}

\subsection{Preliminary Result of In(III)-TMSBr-Catalyzed Cascade Reaction of Diarylalkynes with Acrylates}

More recently, our group has demonstrated that the combined Lewis acid of In(III) salt and TMSBr could effectively activate acrylate component for the reaction with aryl alkynes [49]. Inspired by this activation mode, we envisaged a cascade reaction between diarylalkyne $\mathbf{1}$ and acrylate $\mathbf{2}$ to prepare aryldihydronaphthalene derivatives with this combined Lewis acid via the reaction sequence shown in Scheme 2. Initially, an intramolecular Friedel-Crafts type arylation reaction might occur, to give alkenyl indium species int-1 which will then undergo nucleophilic attack on the activated acrylate 
2 to give the dihydronaphthalene enolate int-2. A further enolate protonation will give the final 1,2-dihydronaphthalene derivative 3.

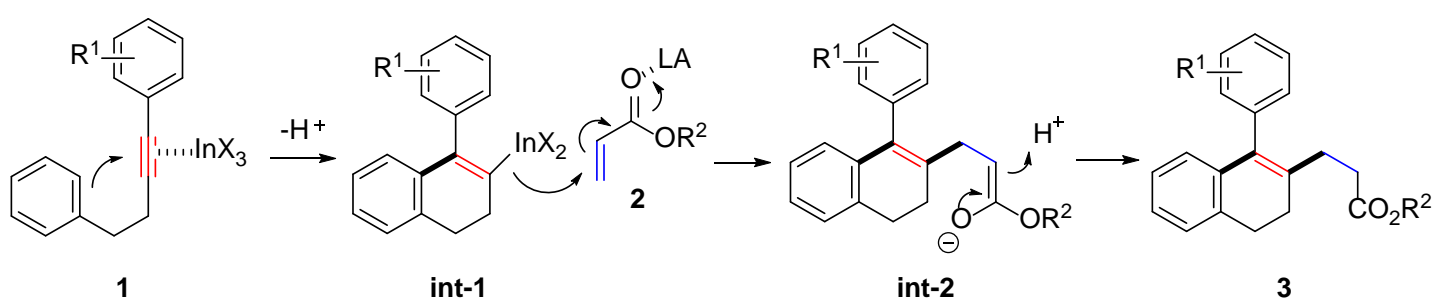

Scheme 2. Proposed In(III)-TMSBr-catalysed cascade reaction of diarylalkynes with acrylates.

To begin with, the model reaction involving but-1-yne-1,4-diyldibenzene (1a) and methyl acrylate (2a) were investigated to probe the proposed cascade reaction. In the presence of $\mathrm{InBr}_{3}$ and $\mathrm{TMSBr}$, a 1,2-dihydronaphthalene derivative 3aa with two propionate motifs was obtained in moderate yield with trace amounts of intractable mixture of 1,2-dihydronaphthalene derivatives containing more propionate motifs (Scheme 3). Interestingly, no 1,2-dihydronaphthalene derivative with a single propionate motif in original proposal could be obtained.

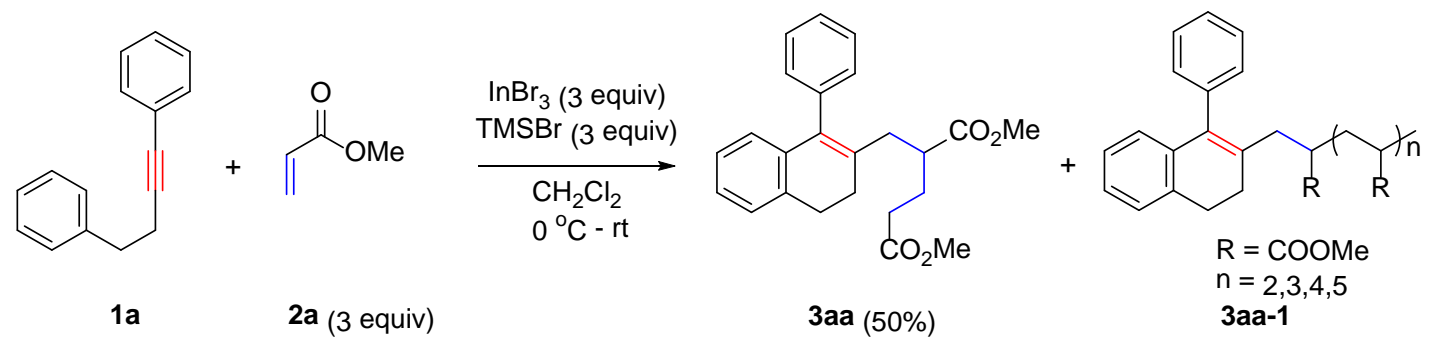

Scheme 3. Initial results of In(III)-TMSBr-catalysed cascade reaction of but-1-yne-1,4-diyldibenzene (1a) with methyl acrylates (2a).

\subsection{Optimization of In(III)-TMSBr-Catalyzed Cascade Reaction of Diarylalkynes with Acrylates}

We subsequently optimized the reaction conditions by using but-1-yne-1,4-diyldibenzene (1a) and methyl acrylate (2a) as model substrates. The results are summarized in Table 1. At the outset, it was found that both In(III) catalyst and TMSBr were indispensable for the efficient progress of this reaction, because the reaction could not take place in the absence of either of them (entries 2-3). Among the different indium catalysts studied (entries 1 and 4-8), $\operatorname{In}(\operatorname{tfacac})_{3}$ was found to exhibit the best catalytic activity to afford the desired product 3aa with $61 \%$ yield (entry 7 ). Other common Lewis acid catalysts (e.g., $\mathrm{AlBr}_{3}$ and $\mathrm{ZnCl}_{2}$, entries 9 and 10) were also screened, which mostly resulted in no product formation. In addition, this reaction was found to proceed only in chlorinated solvents, such as $\mathrm{CH}_{2} \mathrm{Cl}_{2}$ or 1,2-dichloroethane, with the latter giving a higher yield of $70 \%$ (entry 7 vs. entry 11 ). In comparison, when TMSBr was replaced by TMSCl, the product yield eroded significantly to less than $5 \%$ (entry 12). An attempt to decrease the amount of TMSBr or In(tfacac) $)_{3}$ led to lower yields (entries 13-14). Finally, reducing the stoichiometry of methyl acrylate (2a) to only one equivalent resulted in a significantly decreased yield of 3aa, and 1,2-dihydronaphthalene derivative with a single propionate motif remained absent (entry 15). 
Table 1. The In(III)-TMSBr-catalyzed cascade reaction of diarylalkyne 1a with methyl acrylate (2a) ${ }^{\text {a }}$.

catalyst (20 mol\%)
TMSX (3 equiv)

\footnotetext{
a Unless otherwise noted, all reactions were performed with 1 a $(0.4 \mathrm{mmol}), 2 \mathrm{a}(1.2 \mathrm{mmol})$, catalyst $(20 \mathrm{~mol} \%)$, TMSX ( 3 equiv), $0{ }^{\circ} \mathrm{C}-\mathrm{rt}, 2 \mathrm{~h}, \mathrm{~N}_{2}$. $^{\mathrm{b}}$ Isolated yields. ${ }^{\mathrm{c}} 2.0$ equiv of TMSBr was added. ${ }^{\mathrm{d}} 10 \mathrm{~mol} \%$ of In(tfacac) 3 was added. ${ }^{\mathrm{e}} 1.0$ equiv of $\mathbf{2 a}$ was added.
}

\subsection{Substrate Scope of In(III)-TMSBr-Catalysed Cascade Reaction of Diarylalkynes with Acrylates}

With the optimized reaction conditions in hand (Table 1, entry 11), the generality of diarylalkyne substrate scope of this reaction with respect to methyl acrylate (2a) was investigated, and the results are listed in Figure 2a. Various substituted but-1-yne-1,4-diyldibenzene derivatives on the phenyl ring were well suited for this protocol, producing the corresponding product 3 in $47 \%$ to $73 \%$ yields (3aa-ja). Expectedly, substrates with orth-substituent on phenyl ring gave respective products in lower yields than those with meta- or para-substituents (3ga vs. 3ba and 3ha, 3fa vs. 3ia) and no cyclization product could be detected when $\mathbf{1 1}$ was used under the same conditions. Additionally, the incompatibility of 4-phenyl-1-butyne (1k) with the current transformation emphasized the importance of the phenyl ring moiety for this reaction. Finally, a substrate with a strong aryl electron-withdrawing substituent (e.g., 1m) was also unsuitable for this reaction. 


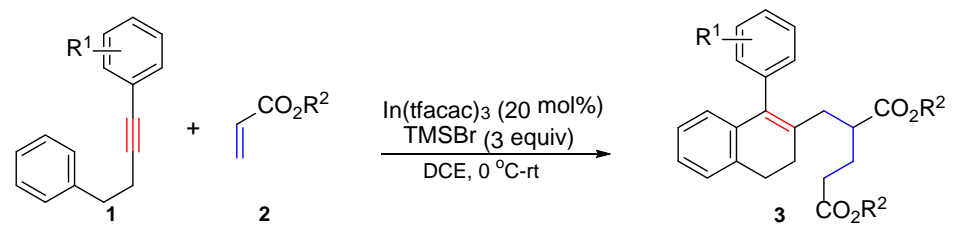

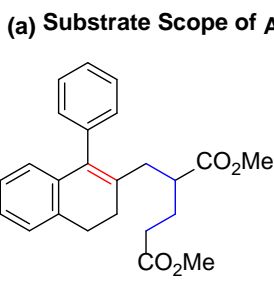<smiles></smiles>
3ea, $65 \%$ $\mathrm{Br}$<smiles>CC(=O)CCC(CC1=C(c2ccc(Br)cc2C)c2ccccc2CC1)C(C)=O</smiles>

3ia, $52 \%$

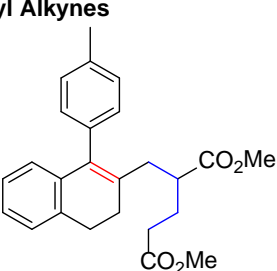
3ba, $73 \%$<smiles>CC(=O)CCC(CC(C)=O)CC1=C(c2ccc(Br)cc2)c2ccccc2CC1</smiles>
3fa, $67 \%$<smiles>CC(=O)CCC(CC(C)=O)CC1=C(c2ccc(-c3ccccc3)cc2)c2ccccc2CC1</smiles>

3ca, $64 \%$<smiles>CC(=O)CCC(CC1=C(c2ccccc2C)c2ccccc2CC1)C(C)=O</smiles>

3ga, $47 \%$<smiles>CC(=O)CCC(CC(C)=O)CC1=C(c2ccc(F)cc2)c2ccccc2CC1</smiles>

3da, $57 \%$<smiles>CC(=O)OCCC(CC1=C(c2cccc(C)c2)c2ccccc2CC1)C(C)=O</smiles>

3ha, $58 \%$

(b) Substrate Scope of Acrylates

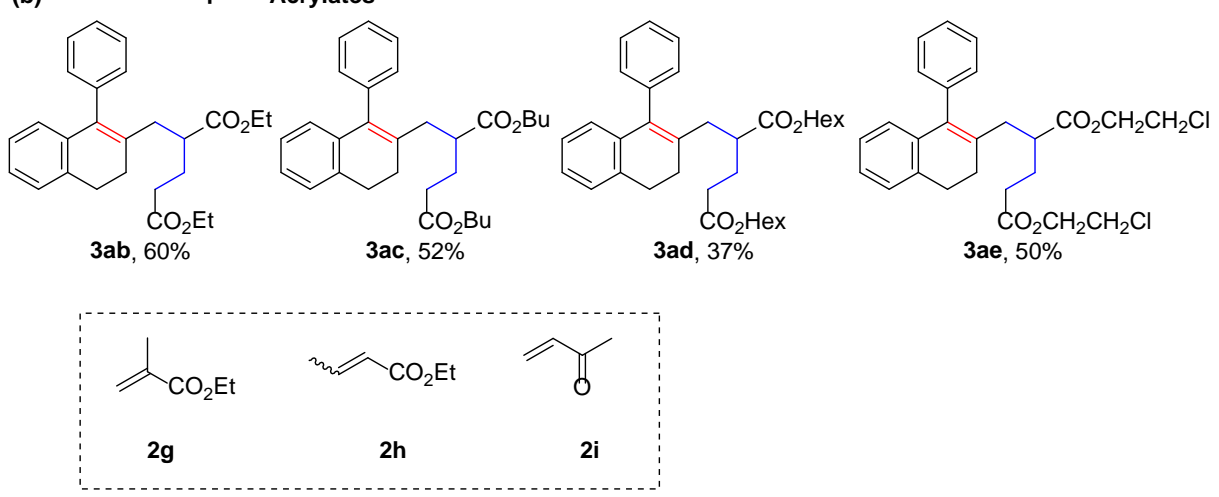

Figure 2. Substrate scope for the In(III)-TMSBr-catalysed cascade reaction of diarylalkyne with acrylates ${ }^{\mathrm{a}, \mathrm{b}}$. a Unless otherwise noted, all reactions were performed with $\mathbf{1}(0.4 \mathrm{mmol}), 2$ (1.2 mmol), In(tfacac) $3(20 \mathrm{~mol} \%), \mathrm{TMSBr}\left(3\right.$ equiv), $0{ }^{\circ} \mathrm{C}-\mathrm{rt}, \mathrm{N}_{2} \cdot{ }^{\mathrm{b}}$ Isolated yields.

With but-1-yne-1,4-diyldibenzene (1a) as the standard coupling partner, next, the scope of acrylate 2 in the present protocol was examined (Figure 2b). In addition to methyl acrylate (3a), acrylates carrying longer $O$-alkyl chains also reacted smoothly to give 1,2-dihydronaphthalene products 3ab-ad, albeit in relatively low yields (37-60\%). Aside from the alkyl chain, reactions of acrylates tethering the chloroethyl group proceeded well to give 3 ae in moderate yield (50\%). However, the use of other olefinic substrates, such as ethyl methacrylate $(2 \mathrm{~g})$, ethyl but-2-enoate $(\mathbf{2 h})$, and but-3-en2-one (2i), could not provide any desired product. 


\section{Discussion}

On the basis of above experimental results and precedent literature reports $[38,39,43,44,49]$, a plausible reaction pathway for this $\mathrm{In}(\mathrm{tfacac})_{3}-\mathrm{TMSBr}$-catalyzed cascade reaction of diarylalkynes with acrylates was put forward in Scheme 4: In(tfacac) $)_{3}$ and TMSBr would first form a combined Lewis acid complex (LA) with heightened acidity than either of them solely; second, an intramolecular Friedel-Crafts type arylation reaction takes place to generate an alkenyl indium species int-1; this is followed by a nucleophilic attack of int-1 onto activated acrylate 2, giving rise to int-2, which subsequently attacks another molecule of acrylate to give int-3; finally, the int-3 is quenched by the proton generated from the first step to furnish the final product 3 .

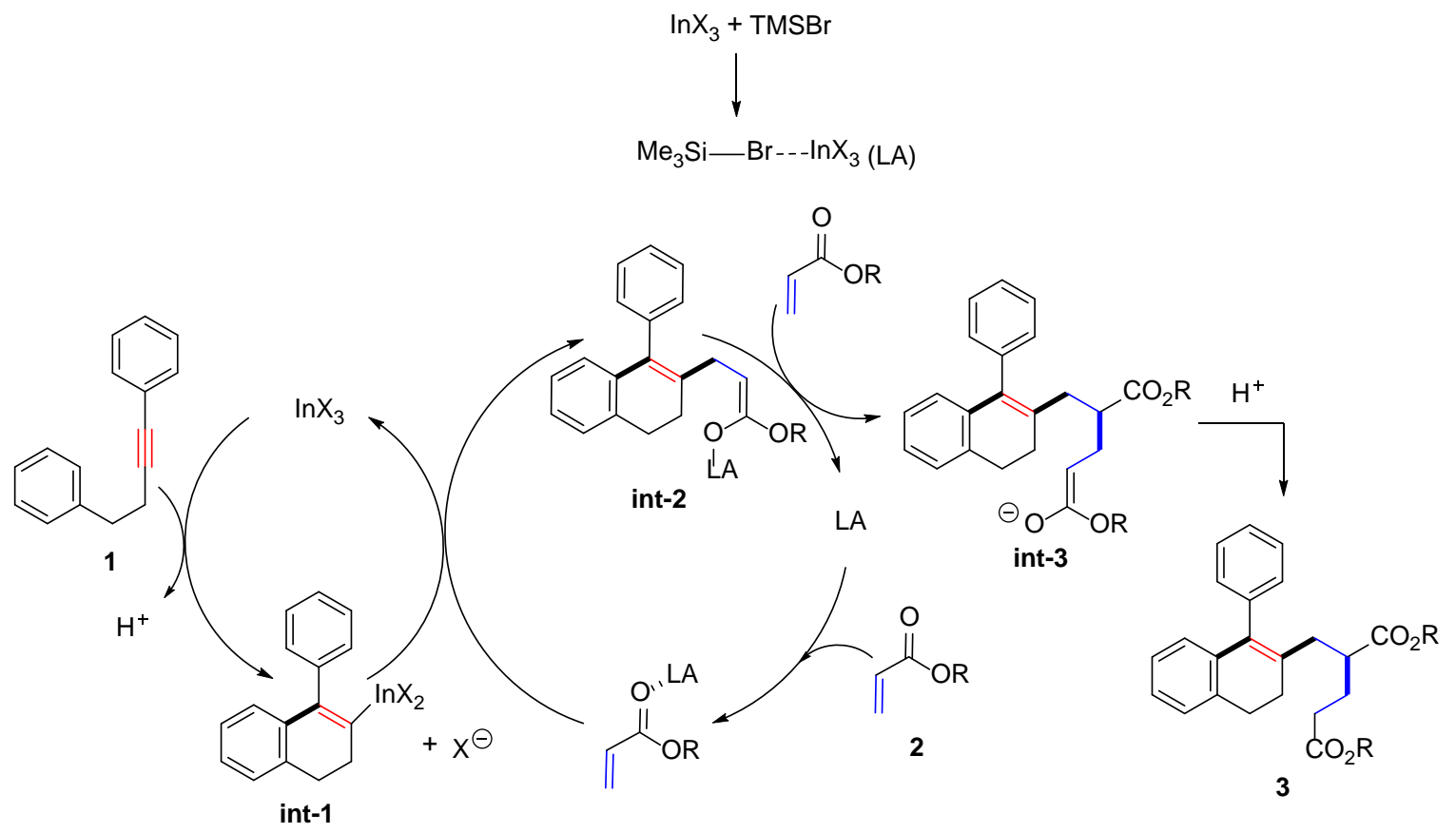

Scheme 4. Plausible reaction mechanism of the In(tfacac) $)_{3}$-TMSBr-catalysed cascade reaction of diarylalkynes with acrylates.

\section{Materials and Methods}

\subsection{General Information}

Unless otherwise noted, all reagents and solvents were purchased from commercial sources and used as received. The TMSBr and $\mathrm{InBr}_{3}$ used was purchased from Sigma-Aldrich, Singapore. The In(tfacac) 3 used was purchased from Strem Chemicals (Newburyport, MA, USA) and its purity is $99 \%$. Thin layer chromatography (TLC) was used to monitor the reaction progress on Merck (Frankfurter Strasse, Darmstadt, Germany) 60 F254 precoated silica gel plates (0.2 mm thickness). TLC spots were visualized by UV-light irradiation on a Spectro line model ENF-24061/F (Spectroline, Westbury, NY, USA) at $254 \mathrm{~nm}$. Anther visualization method was staining with a basic solution of potassium permanganate or acidic solution of ceric molybdate, followed by heating. Flash column chromatography was performed using Merck silica gel 60 (Frankfurter Strasse, Darmstadt, Germany) with analytical grade solvents as eluents. ${ }^{1} \mathrm{H}-\mathrm{NMR},{ }^{13} \mathrm{C}-\mathrm{NMR}$, and $2 \mathrm{D} \mathrm{NMR}$ spectra were recorded using Bruker Avance $400 \mathrm{MHz}$ spectrometers (Bruker Corporation, Billerica, MA, USA). Corresponding chemical shifts are reported in ppm downfield relative to TMS and were referenced to the signal of chloroform-d $(\delta=7.26$, singlet). Multiplicities were given as: $\mathrm{s}=$ singlet, $\mathrm{d}=\mathrm{doublet}$, $\mathrm{t}=\mathrm{triplet}$, $\mathrm{q}=$ quartet, $\mathrm{m}=$ multiplet, brs = broad singlet, $\mathrm{dd}=$ doublet of doublets, and $\mathrm{td}=$ triplet of doublets. Values of coupling constants are reported as $J$ in $\mathrm{Hz}$. 


\subsection{General Procedure for In(III)-TMSBr-Catalysed Cascade Reaction of Diarylalkyne with Acrylates}

A dry reaction tube was charged with aryl alkyne $\mathbf{1}(0.4 \mathrm{mmol})$, acrylate $\mathbf{2}(1.2 \mathrm{mmol})$, indium(III) trifluoroacetylacetonate $\left(\operatorname{In}(\text { tfacac) })_{3}, 20 \mathrm{~mol} \%, 0.08 \mathrm{mmol}, 45.9 \mathrm{mg}\right.$ ) and DCE (1 mL) under $\mathrm{N}_{2}$ atmosphere at $0{ }^{\circ} \mathrm{C}$. Bromotrimethylsilane (TMSBr, 3 equiv, $1.2 \mathrm{mmol}, 183.6 \mathrm{mg}$ ) was added and the reaction mixture was stirred at room temperature for $2 \mathrm{~h}$. Upon completion of the reaction as indicated by TLC analysis, the residue was directly purified by flash column chromatography on silica gel (eluent: hexane/ethyl acetate 10:1) to afford the desired product 3.

\subsection{Product Characterization}

Dimethyl 2-((1-phenyl-3,4-dihydronaphthalen-2-yl)methyl)pentanedioate (3aa). Colorless oil, $106.2 \mathrm{mg}$, $0.281 \mathrm{mmol}, 70 \%$ yield. ${ }^{1} \mathrm{H}-\mathrm{NMR}\left(400 \mathrm{MHz}, \mathrm{CDCl}_{3}\right): \delta(\mathrm{ppm}) 7.45-7.41(\mathrm{~m}, 2 \mathrm{H}), 7.38-7.34(\mathrm{~m}, 1 \mathrm{H})$, 7.18-7.10 (m, 4H), 7.05-7.02 (m, 1H), $6.58(\mathrm{~d}, J=7.5 \mathrm{~Hz}, 1 \mathrm{H}), 3.67(\mathrm{~s}, 3 \mathrm{H}), 3.65(\mathrm{~s}, 3 \mathrm{H}), 2.91(\mathrm{t}, J=7.7 \mathrm{~Hz}$, 2H), 2.66-2.61 (m, 1H), 2.44-2.31 (m, 4H), 2.22-2.18 (m, 2H), 1.79-1.76 (m, 2H); ${ }^{13} \mathrm{C}-\mathrm{NMR}(100 \mathrm{MHz}$, $\left.\mathrm{CDCl}_{3}\right): \delta$ (ppm) 175.4, 173.3, 139.2, 136.7, 136.3, 135.2, 134.3, 130.2, 128.4, 127.0, 126.9, 126.4, 126.2, $125.8,51.6,51.5,43.5,37.0,31.6,28.4,27.4,26.7$.

Dimethyl 2-((1-(p-tolyl)-3,4-dihydronaphthalen-2-yl)methyl)pentanedioate (3ba). Colorless oil, $114.5 \mathrm{mg}$, $0.292 \mathrm{mmol}, 73 \%$ yield. ${ }^{1} \mathrm{H}-\mathrm{NMR}\left(400 \mathrm{MHz}, \mathrm{CDCl}_{3}\right): \delta(\mathrm{ppm}) 7.24-7.22(\mathrm{~m}, 2 \mathrm{H}), 7.17-7.12(\mathrm{~m}, 1 \mathrm{H})$, 7.11-7.08 (m, 1H), 7.05-7.01 (m, 3H), $6.60(\mathrm{~d}, J=7.0 \mathrm{~Hz}, 1 \mathrm{H}), 3.67(\mathrm{~s}, 3 \mathrm{H}), 3.65(\mathrm{~s}, 3 \mathrm{H}), 2.89(\mathrm{t}, J=7.8 \mathrm{~Hz}$, $2 \mathrm{H}), 2.66-2.62(\mathrm{~m}, 1 \mathrm{H}), 2.44(\mathrm{~s}, 3 \mathrm{H}), 2.43-2.35(\mathrm{~m}, 4 \mathrm{H}), 2.22-2.18(\mathrm{~m}, 2 \mathrm{H}), 1.79-1.74(\mathrm{~m}, 2 \mathrm{H}) ;{ }^{13} \mathrm{C}-\mathrm{NMR}$ $\left(100 \mathrm{MHz}_{\mathrm{CDCl}}\right): \delta(\mathrm{ppm}) 175.5,173.3,136.8,136.4,136.2,136.0,135.2,134.2,130.1,129.1,127.0,126.3$, $126.2,125.9,51.6,51.5,43.4,37.0,31.6,28.4,27.4,26.6,21.2$.

Dimethyl 2-((1-([1,1'-biphenyl]-4-yl)-3,4-dihydronaphthalen-2-yl)methyl)pentanedioate (3ca). Colorless oil, $116.3 \mathrm{mg}, 0.256 \mathrm{mmol}, 64 \%$ yield. ${ }^{1} \mathrm{H}-\mathrm{NMR}\left(400 \mathrm{MHz} \mathrm{CDCl}_{3}\right): \delta(\mathrm{ppm}) 7.71-7.66(\mathrm{~m}, 4 \mathrm{H}), 7.51-7.47(\mathrm{~m}, 2 \mathrm{H})$, 7.44-7.37 (m, 2H), 7, 23 (brs, 2H), 7.15-7.11 (m, 1H), 7.08-7.04 (m, 1H), $6.67(\mathrm{~d}, J=7.6 \mathrm{~Hz}, 1 \mathrm{H}), 3.66(\mathrm{~s}, 3 \mathrm{H})$, $3.65(\mathrm{~s}, 3 \mathrm{H}), 2.94-2.90(\mathrm{~m}, 2 \mathrm{H}), 2.70-2.66(\mathrm{~m}, 1 \mathrm{H}), 2.50-2.41(\mathrm{~m}, 4 \mathrm{H}), 2.25-2.19(\mathrm{~m}, 2 \mathrm{H}), 1.83-1.77(\mathrm{~m}, 2 \mathrm{H})$; ${ }^{13} \mathrm{C}-\mathrm{NMR}\left(100 \mathrm{MHz}, \mathrm{CDCl}_{3}\right): \delta$ (ppm) 175.5, 173.3, 140.9, 139.6, 138.2, 136.7, 135.9, 135.2, 134.5, $130.7(\times 2)$, $128.8,127.3,127.1(\times 2), 126.4,126.2,125.9,51.7,51.6,43.5,37.0,31.6,28.4,27.4,26.6$.

Dimethyl 2-((1-(4-fluorophenyl)-3,4-dihydronaphthalen-2-yl)methyl)pentanedioate (3da). Colorless oil, $90.3 \mathrm{mg}$, $0.228 \mathrm{mmol}, 57 \%$ yield. ${ }^{1} \mathrm{H}-\mathrm{NMR}\left(400 \mathrm{MHz}, \mathrm{CDCl}_{3}\right): \delta(\mathrm{ppm}) 7.18-7.16(\mathrm{~m}, 1 \mathrm{H}), 7.14-7.10(\mathrm{~m}, 5 \mathrm{H})$, 7.07-7.03 (m, 1H), 6.56-6.54 (d, J = 7.5 Hz, 1H), $3.68(\mathrm{~s}, 3 \mathrm{H}), 3.65(\mathrm{~s}, 3 \mathrm{H}), 2.90(\mathrm{t}, J=7.9 \mathrm{~Hz}, 2 \mathrm{H})$, 2.66-2.61 (m, 1H), 2.44-2.29 (m, 4H), 2.24-2.19 (m, 2H), 1.80-1.73 (m, 2H); ${ }^{13} \mathrm{C}-\mathrm{NMR}\left(100 \mathrm{MHz}, \mathrm{CDCl}_{3}\right)$ : $\delta(\mathrm{ppm}) 175.3,173.3,161.9\left(\mathrm{~d}, J_{\mathrm{C}-\mathrm{F}}=243.7 \mathrm{~Hz}\right), 136.5,135.3,135.2,134.9\left(\mathrm{~d}, J_{\mathrm{C}-\mathrm{F}}=4.0 \mathrm{~Hz}\right), 134.8,131.8\left(\mathrm{~d}, J_{\mathrm{C}-\mathrm{F}}\right.$ $=7.8 \mathrm{~Hz}), 127.1,126.5,126.2,125.7,115.3\left(\mathrm{~d}, J_{\mathrm{C}-\mathrm{F}}=21.1 \mathrm{~Hz}\right), 51.7,51.6,43.4,37.0,31.5,28.3,27.4,26.7$.

Dimethyl 2-((1-(4-chlorophenyl)-3,4-dihydronaphthalen-2-yl)methyl)pentanedioate (3ea). Colorless oil, $107.1 \mathrm{mg}$, $0.26 \mathrm{mmol}, 65 \%$ yield. ${ }^{1} \mathrm{H}-\mathrm{NMR}\left(400 \mathrm{MHz} \mathrm{CDCl}_{3}\right): \delta(\mathrm{ppm}) 7.61-7.59(\mathrm{~m}, 2 \mathrm{H}), 7.22-7.14(\mathrm{~m}, 3 \mathrm{H})$, 7.10-7.06 (m, 2H), $6.58(\mathrm{~d}, J=7.6 \mathrm{~Hz}, 1 \mathrm{H}), 3.72(\mathrm{~s}, 3 \mathrm{H}), 3.69(\mathrm{~s}, 3 \mathrm{H}), 2.93(\mathrm{t}, J=7.8 \mathrm{~Hz}, 2 \mathrm{H}), 2.71-2.67(\mathrm{~m}, 1 \mathrm{H})$, 2.48-2.41 (m, 4H), 2.37-2.32 (m, 2H), 1.83-1.77 (m, 2H); ${ }^{13} \mathrm{C}-\mathrm{NMR}\left(100 \mathrm{MHz}, \mathrm{CDCl}_{3}\right): \delta(\mathrm{ppm})$ 175.3, 173.2, 138.1, 136.2, 135.2, 135.1, 134.8, 132.0, 131.6, 127.1, 126.6, 126.3, 125.7, 121.0, 51.7, 51.6, 43.3, 37.0, 31.5, 28.3, 27.3, 26.7.

Dimethyl 2-((1-(4-bromophenyl)-3,4-dihydronaphthalen-2-yl)methyl)pentanedioate (3fa). Colorless oil, $122.2 \mathrm{mg}$, $0.268 \mathrm{mmol}, 67 \%$ yield. ${ }^{1} \mathrm{H}-\mathrm{NMR}\left(400 \mathrm{MHz}, \mathrm{CDCl}_{3}\right): \delta(\mathrm{ppm}) 7.41-7.39(\mathrm{~m}, 2 \mathrm{H}), 7.17-7.02(\mathrm{~m}, 5 \mathrm{H})$, $6.54(\mathrm{~d}, J=7.6 \mathrm{~Hz}, 1 \mathrm{H}), 3.67(\mathrm{~s}, 3 \mathrm{H}), 3.65(\mathrm{~s}, 3 \mathrm{H}), 2.89(\mathrm{t}, J=7.9 \mathrm{~Hz}, 2 \mathrm{H}), 2.68-2.61(\mathrm{~m}, 1 \mathrm{H}), 2.43-2.26(\mathrm{~m}, 4 \mathrm{H})$, 2.24-2.19 (m, 2H), 1.79-1.75 (m, 2H); ${ }^{13} \mathrm{C}-\mathrm{NMR}\left(100 \mathrm{MHz}, \mathrm{CDCl}_{3}\right)$ : $\delta$ (ppm) 175.3, 173.2, 137.6, 136.3, 135.1, $134.9,132.9,131.7,128.7$ (×2), 127.1, 126.6, 126.3, 125.7, 51.7, 51.6, 43.3, 37.0, 31.5, 28.3, 27.3, 26.7.

Dimethyl 2-((1-(o-tolyl)-3,4-dihydronaphthalen-2-yl)methyl)pentanedioate (3ga). Colorless oil, $73.7 \mathrm{mg}$, $0.188 \mathrm{mmol}, 47 \%$ yield. ${ }^{1} \mathrm{H}-\mathrm{NMR}\left(400 \mathrm{MHz}, \mathrm{CDCl}_{3}\right): \delta(\mathrm{ppm}) 7.28-7.22(\mathrm{~m}, 3 \mathrm{H}), 7.18-7.17(\mathrm{~m}, 1 \mathrm{H})$, 7.12-7.08 (m, 2H), 7.03-7.00 (m, 1H), $6.49(\mathrm{~d}, J=7.4 \mathrm{~Hz}, 1 \mathrm{H}), 3.66(\mathrm{~s}, 3 \mathrm{H}), 3.65(\mathrm{~s}, 3 \mathrm{H}), 2.92-2.90(\mathrm{~m}, 2 \mathrm{H})$, 
2.64-2.61 (m, 1H), 2.46-2.29 (m, 4H), 2.19-2.15 (m, 2H), $2.05(\mathrm{~s}, 3 \mathrm{H}), 1.79-1.72(\mathrm{~m}, 2 \mathrm{H}) ;{ }^{13} \mathrm{C}-\mathrm{NMR}(100 \mathrm{MHz}$, $\left.\mathrm{CDCl}_{3}\right): \delta$ (ppm) 175.7, 173.3, 138.3, 136.8, 135.8, 135.4, 135.1, 134.2, 130.6, 130.4, 130.1, 127.3, 127.0, 126.4, $125.8,125.1,51.6,51.5,43.3,36.8,31.5,28.4,27.1,26.5,19.3$.

Dimethyl 2-((1-(m-tolyl)-3,4-dihydronaphthalen-2-yl)methyl)pentanedioate (3ha). Colorless oil, $90.9 \mathrm{mg}$, $0.232 \mathrm{mmol}, 58 \%$ yield. ${ }^{1} \mathrm{H}-\mathrm{NMR}\left(400 \mathrm{MHz}, \mathrm{CDCl}_{3}\right): \delta(\mathrm{ppm}) 7.32-7.30(\mathrm{~m}, 1 \mathrm{H}), 7.17-7.15(\mathrm{~m}, 2 \mathrm{H})$, 7.12-7.08 (m, 1H), 7.05-7.01 (m, 1H), 6.97-6.93 (m, 2H), $6.59(\mathrm{~d}, J=7.5 \mathrm{~Hz}, 1 \mathrm{H}), 3.66(\mathrm{~s}, 3 \mathrm{H}), 3,65(\mathrm{~s}, 3 \mathrm{H})$, 2.91-2.87 (m, 2H), 2.65-2.62 (m, 1H), 2.43-2.34 (m, 4H), $2.39(\mathrm{~s}, 3 \mathrm{H}), 2.22-2.17(\mathrm{~m}, 2 \mathrm{H}), 1.79-1.76(\mathrm{~m}, 2 \mathrm{H})$; ${ }^{13} \mathrm{C}-\mathrm{NMR}\left(100 \mathrm{MHz}, \mathrm{CDCl}_{3}\right)$ : $\delta$ (ppm) 175.6, 173.3, 139.0, 136.7, 136.3, 135.1, 134.1, 130.8, 128.2, 127.6 (×2), 127.2, 127.0, 126.3, 126.2, 125.9, 51.6, 51.5, 43.4, 36.9, 31.6, 28.4, 27.3, 26.6, 21.5.

Dimethyl 2-((1-(4-bromo-2-methylphenyl)-3,4-dihydronaphthalen-2-yl)methyl)pentanedioate (3ia). Colorless oil, $97.8 \mathrm{mg}, 0.208 \mathrm{mmol}, 52 \%$ yield. ${ }^{1} \mathrm{H}-\mathrm{NMR}\left(400 \mathrm{MHz}, \mathrm{CDCl}_{3}\right): \delta(\mathrm{ppm}) 7.44(\mathrm{~s}, 1 \mathrm{H}), 7.38(\mathrm{~d}, J=8.1 \mathrm{~Hz}, 1 \mathrm{H})$, $7.17(\mathrm{~d}, J=7.1 \mathrm{~Hz}, 1 \mathrm{H}), 7.13-7.10(\mathrm{~m}, 1 \mathrm{H}), 7.04-7.00(\mathrm{~m}, 1 \mathrm{H}), 6.97(\mathrm{~d}, J=8.1 \mathrm{~Hz}, 1 \mathrm{H}), 6.45(\mathrm{~d}, J=7.4 \mathrm{~Hz}$, $1 \mathrm{H}), 3.67(\mathrm{~s}, 3 \mathrm{H}), 3.66(\mathrm{~s}, 3 \mathrm{H}), 2.92-2.88(\mathrm{~m}, 2 \mathrm{H}), 2.64-2.62(\mathrm{~m}, 1 \mathrm{H}), 2.46-2.34(\mathrm{~m}, 4 \mathrm{H}), 2.22-2.11(\mathrm{~m}, 2 \mathrm{H})$, $2.03(\mathrm{~s}, 3 \mathrm{H}), 1.80-1.70(\mathrm{~m}, 2 \mathrm{H}) ;{ }^{13} \mathrm{C}-\mathrm{NMR}\left(100 \mathrm{MHz}, \mathrm{CDCl}_{3}\right): \delta(\mathrm{ppm}) 175.5,173.2,139.4,137.3,135.3,135.1$, 134.8, 134.3, 133.0, 132.1, 129.0, 127.2, 126.6, 126.4, 124.9, 121.1, 51.7, 51.6, 43.2, 36.9, 31.5, $28.3(\times 2), 27.0,26.5$.

Dimethyl 2-((1-(3,5-dimethylphenyl)-3,4-dihydronaphthalen-2-yl)methyl)pentanedioate (3ja). Colorless oil, $89.4 \mathrm{mg}, 0.220 \mathrm{mmol}, 55 \%$ yield. ${ }^{1} \mathrm{H}-\mathrm{NMR}\left(400 \mathrm{MHz}, \mathrm{CDCl}_{3}\right): \delta(\mathrm{ppm}) 7.17-7.15(\mathrm{~m}, 1 \mathrm{H}), 7.12-7.08(\mathrm{~m}, 1 \mathrm{H})$, 7.06-7.02 (m, 1H), $6.98(\mathrm{~s}, 1 \mathrm{H}), 6.78(\mathrm{~s}, 1 \mathrm{H}), 6.73(\mathrm{~s}, 1 \mathrm{H}), 6.62(\mathrm{~d}, J=7.5 \mathrm{~Hz}, 1 \mathrm{H}), 3.67(\mathrm{~s}, 3 \mathrm{H}), 3.66(\mathrm{~s}, 3 \mathrm{H})$, 2.91-2.86 (m, 2H), 2.66-2.61 (m, 1H), 2.44-2.37 (m, 4H), $2.35(\mathrm{~s}, 6 \mathrm{H}), 2.24-2.14(\mathrm{~m}, 2 \mathrm{H}), 1.81-1.75(\mathrm{~m}, 2 \mathrm{H})$; ${ }^{13} \mathrm{C}-\mathrm{NMR}\left(100 \mathrm{MHz}, \mathrm{CDCl}_{3}\right): \delta$ (ppm) 175.7, 173.3, 139.0, 137.7, 136.8, 136.4, 135.1, 133.9, 128.4, 127.9, 126.9, $126.2,126.1,125.9,51.6,51.5,43.5,37.0,31.6,28.4,27.3,26.6,21.3$.

Diethyl 2-((1-phenyl-3,4-dihydronaphthalen-2-yl)methyl)pentanedioate (3ab). Colorless oil, $97.5 \mathrm{mg}, 0.240 \mathrm{mmol}$, $60 \%$ yield. ${ }^{1} \mathrm{H}-\mathrm{NMR}\left(400 \mathrm{MHz}, \mathrm{CDCl}_{3}\right): \delta(\mathrm{ppm}) 7.44-7.40(\mathrm{~m}, 2 \mathrm{H}), 7.37-7.33(\mathrm{~m} \mathrm{1H}), 7.18-7.16(\mathrm{~m}, 3 \mathrm{H})$, $7.12-7.09(\mathrm{~m}, 1 \mathrm{H}), 7.05-7.01(\mathrm{~m}, 1 \mathrm{H}), 6.58(\mathrm{~d}, J=7.6 \mathrm{~Hz}, 1 \mathrm{H}), 4.17-4.08(\mathrm{~m}, 4 \mathrm{H}), 2.90(\mathrm{t}, J=8.3 \mathrm{~Hz}, 1 \mathrm{H})$, 2.65-2.58 (m, 1H), 2.43-2.30 (m, 4H), 2.21-2.14 (m, 2H), 1.79-1.74 (m, 2H), 1.29-1.27 (m, 6H); ${ }^{13} \mathrm{C}-\mathrm{NMR}$ $\left(100 \mathrm{MHz}, \mathrm{CDCl}_{3}\right): \delta$ (ppm) 175.1, 172.9, 139.2, 136.7, 136.2, 135.2, 134.5, 130.3, 128.4, 127.0, 126.8, 126.3, $126.2,125.8,60.4,60.3,43.5,37.0,31.8,28.4,27.4,26.8,14.2$.

Dibutyl 2-((1-phenyl-3,4-dihydronaphthalen-2-yl)methyl)pentanedioate(3ac). Colorless oil, $96.2 \mathrm{mg}$, $0.208 \mathrm{mmol}, 52 \%$ yield. ${ }^{1} \mathrm{H}-\mathrm{NMR}\left(400 \mathrm{MHz}, \mathrm{CDCl}_{3}\right): \delta(\mathrm{ppm}) 7.44-7.40(\mathrm{~m}, 2 \mathrm{H}), 7.37-7.33(\mathrm{~m}, 1 \mathrm{H})$, 7.17-7.09 (m, 4H), 7.05-7.01 (m, 1H), $6.58(\mathrm{~d}, J=7.0 \mathrm{~Hz}, 1 \mathrm{H}), 4.07-4.03(\mathrm{~m}, 4 \mathrm{H}), 2.91(\mathrm{t}, J=8.1 \mathrm{~Hz}$, $2 \mathrm{H}), 2.66-2.57(\mathrm{~m}, 1 \mathrm{H}), 2.47-2.30(\mathrm{~m}, 4 \mathrm{H}), 2.21-2.12(\mathrm{~m}, 2 \mathrm{H}), 1.81-1.73(\mathrm{~m}, 2 \mathrm{H}), 1.65-1.54(\mathrm{~m}, 4 \mathrm{H})$, $1.42-1.29(\mathrm{~m}, 4 \mathrm{H}), 0.97-0.89(\mathrm{~m}, 6 \mathrm{H}) ;{ }^{13} \mathrm{C}-\mathrm{NMR}\left(100 \mathrm{MHz}, \mathrm{CDCl}_{3}\right): \delta$ (ppm) 175.1, 173.0, 139.2, 136.7, 136.2, 135.1, 134.4, 130.3, 128.4, 127.0, 126.8, 126.3, 126.2, 125.8, 64.4, 64.3, 43.5, 37.0, 31.9, 30.7, 30.6, 28.4, $27.4,26.8,19.1(\times 2), 13.7,13.6$.

Dihexyl 2-((1-phenyl-3,4-dihydronaphthalen-2-yl)methyl)pentanedioate(3ad). Colorless oil, $66.1 \mathrm{mg}$, $0.148 \mathrm{mmol}, 37 \%$ yield. ${ }^{1} \mathrm{H}-\mathrm{NMR}\left(400 \mathrm{MHz}, \mathrm{CDCl}_{3}\right): \delta(\mathrm{ppm}) 7.44-7.40(\mathrm{~m}, 2 \mathrm{H}), 7.37-7.35(\mathrm{~m}, 1 \mathrm{H})$, 7.17-7.16 (m, 3H), 7.12-7.08 (m, 1H), 7.05-7.01 (m, 1H), $6.57(\mathrm{~d}, J=7.5 \mathrm{~Hz}, 1 \mathrm{H}), 4.09-4.02(\mathrm{~m}, 4 \mathrm{H})$, 2.92-2.88 (m, 2H), 2.64-2.62 (m, 1H), 2.45-2.32 (m, 4H), 2.22-2.16 (m, 2H), 1.79-1.71 (m, 2H), $1.65-1.56(\mathrm{~m}, 6 \mathrm{H}), 1.32-1.28(\mathrm{~m}, 10 \mathrm{H}), 0.93-0.87(\mathrm{~m}, 6 \mathrm{H}) ;{ }^{13} \mathrm{C}-\mathrm{NMR}\left(100 \mathrm{MHz}, \mathrm{CDCl}_{3}\right): \delta(\mathrm{ppm})$ 175.1, 173.0, 139.2, 136.7, 136.2, 135.1, 134.4, 130.3, 128.4, 127.0, 126.8, 126.3, 126.2, 125.8, 64.7, 64.6, 43.5, 37.0, $31.8,31.4,31.3,28.6,28.5,28.4,27.4,26.7,25.6(\times 2), 22.5,22.4,14.0(\times 2)$.

Bis(2-chloroethyl) 2-((1-phenyl-3,4-dihydronaphthalen-2-yl)methyl)pentanedioate (3ae). Colorless oil, $89.2 \mathrm{mg}$, $0.200 \mathrm{mmol}, 50 \%$ yield. ${ }^{1} \mathrm{H}-\mathrm{NMR}\left(400 \mathrm{MHz}, \mathrm{CDCl}_{3}\right): \delta(\mathrm{ppm}) 7.45-7.43(\mathrm{~m}, 2 \mathrm{H}), 7.38-7.36(\mathrm{~m}, 1 \mathrm{H})$, 7.18-7.10 (m, 4H), 7.06-7.02 (m, 1H), $6.58(\mathrm{~d}, J=7.6 \mathrm{~Hz}, 1 \mathrm{H}), 4.35-4.29(\mathrm{~m}, 4 \mathrm{H}), 3.70-3.63(\mathrm{~m}, 4 \mathrm{H})$, 2.94-2.89 (m, 2H), 2.73-2.67 (m, 1H), 2.50-2.33 (m, 4H), 2.31-2.26 (m, 2H), 1.84-1.79 (m, 2H); ${ }^{13} \mathrm{C}-\mathrm{NMR}$ $\left(100 \mathrm{MHz}, \mathrm{CDCl}_{3}\right.$ ): $\delta$ (ppm) 174.6, 172.4, 139.1, 136.6, 136.4, 135.1, 134.0, 130.2, 128.4, 127.0, 126.9, 126.4, $126.2,125.9,64.0,63.9,43.2,41.5,41.4,36.9,31.5,28.4,27.4,26.4$. 
Product characterization data, and ${ }^{1} \mathrm{H}-$ and ${ }^{13} \mathrm{C}-\mathrm{NMR}$ spectra are available from supplementary material.

\section{Conclusions}

In a nutshell, we described an efficient method to assemble aryldihydronaphthalene derivatives via the cascade reaction of diarylalkynes with acrylates employing the catalysis of a combined Lewis acid system formed from In(III) salt and TMSBr. Both indium(III) and TMSBr are indispensable for the efficient progress of the reaction. In most cases, the reaction proceeded efficiently to afford the corresponding aryldihydronaphthalene derivatives in moderate to good yields. With reference to current experimental observations and literature reports, a possible mechanistic pathway for this reaction was also provided.

Supplementary Materials: Supplementary materials are available online: general experimental procedures, product characterization data, and ${ }^{1} \mathrm{H}$ - and ${ }^{13} \mathrm{C}-\mathrm{NMR}$ spectra.

Acknowledgments: We are grateful to Nanjing Tech University, the SICAM Fellowship from the Jiangsu National Synergetic Innovation Center for Advanced Materials, Nanyang Technological University, Singapore Ministry of Education Academic Research Fund (Tier 1: MOE2015-T1-001-070 (RG5/15) and MOE2014-T1-001-102 (RG9/14)), and the Singapore National Research Foundation (NRF2015NRF-POC001-024) for generous financial support. W.W.Z. thanks BGI for financial support.

Author Contributions: W.W.Z. and T.-P.L. conceived and designed the experiments; L.S. and Q.-C.Z. performed the experiments; L.S. and Z.L.S wrote the paper; and W.W.Z. and L.S. analysed the data.

Conflicts of Interest: The authors declare no conflict of interest.

\section{References}

1. James, M.J.; O’Brien, P.; Taylor, R.J.K.; Unsworth, W.P. Synthesis of spirocyclic indolenines. Chem. Eur. J. 2016, 22, 2856-2881. [CrossRef] [PubMed]

2. Nicolaou, K.C.; Edmonds, D.J.; Bulger, P.G. Cascade reactions in total synthesis. Angew. Chem. Int. Ed. 2006, 45, 7134-7186. [CrossRef] [PubMed]

3. Pan, J.-Y.; Chen, S.-L.; Yang, M.-H.; Wu, J.; Sinkkonen, J.; Zou, K. An update on lignans: Natural products and synthesis. Nat. Prod. Rep. 2009, 26, 1251-1292. [CrossRef] [PubMed]

4. Teponno, R.B.; Kusari, S.; Spiteller, M. Recent advances in research on lignans and neolignans. Nat. Prod. Rep. 2016, 33, 1044-1092. [CrossRef] [PubMed]

5. Yang, X.; Reinhold, A.R.; Rosati, R.L.; Liu, K.K.C. Enzyme-catalyzed asymmetric deacylation for the preparation of Lasofoxifene (CP-336156), a selective estrogen receptor modulator. Org. Lett. 2000, 2, 4025-4027. [CrossRef] [PubMed]

6. Haq, A.-U.; Malik, A.; Anis, I.; Khan, S.B.; Ahmed, E.; Ahmed, Z.; Nawas, S.A.; Choudhary, M.I. Enzymes inhibiting lignans from Vitex Negundo. Chem. Pharm. Bull. 2004, 52, 1269-1272. [CrossRef]

7. Kocsis, L.S.; Brummond, K.M. Intramolecular dehydro-Diels-Alder reaction affords selective entry to arylnaphthalene or aryldihydronaphthalene lignans. Org. Lett. 2014, 16, 4158-4161. [CrossRef] [PubMed]

8. Magoulas, G.E.; Papaioannou, D. Bioinspired syntheses of dimeric hydroxycinnamic acids (lignans) and hybrids, using phenol oxidative coupling as key reaction, and medicinal significance thereof. Molecules 2014, 19, 19769-19835. [CrossRef] [PubMed]

9. Silva, L.F.; Siqueira, F.A.; Pedrozo, E.C.; Vieira, F.Y.M.; Doriguetto, A.C. Iodine(III)-promoted ring contraction of 1,2-dihydronaphthalenes: Adiastereoselective total synthesis of ( \pm )-Indatraline. Org. Lett. 2007, 9, 1433-1436. [CrossRef] [PubMed]

10. Scribner, A.W.; Haroutounian, S.A.; Carlson, K.E.; Katzenellenbogen, J.A. 1-Aryl-2-pyridyl-3,4dihydronaphthalenes: photofluorogenic ligands for the estrogen receptor. J. Org. Chem. 1997, 62, 1043-1057. [CrossRef]

11. Marieke, V.; Iris, A.; Christiane, S.; Ursula, M.-V.; Klaus, B.; Sandrine, M.-O.; Rolf, W.H. Synthesis and evaluation of heteroaryl-substituted dihydronaphthalenes and indenes: Potent and selective inhibitors of aldosterone synthase (CYP11B2) for the treatment of congestive heart failure and myocardial fibrosis. J. Med. Chem. 2006, 49, 2222-2231. [CrossRef] 
12. Bandini, M.; Emer, E.; Tommasi, S.; Umani-Ronchi, A. Innovative catalytic protocols for the ring-closing Friedel-Crafts-typealkylation and alkenylation of arenes. Eur. J. Org. Chem. 2006, 3527-3544. [CrossRef]

13. Kitamura, T. Transition-metal-catalyzed hydroarylation reactions of alkynes through direct functionalization of C-H bonds: a convenient tool for organic synthesis. Eur. J. Org. Chem. 2009, 1111-1125. [CrossRef]

14. Yamamoto, Y. Synthesis of heterocycles via transition-metal catalyzed hydroarylation of alkynes. Chem. Soc. Rev. 2014, 43, 1575-1600. [CrossRef] [PubMed]

15. Namyslo, J.C.; Storsberg, J.; Klinge, J.; Gärtner, C.; Yao, M.-L.; Ocal, N.; Kaufmann, D.E. The hydroarylation reaction-Scope and limitations. Molecules 2010, 15, 3402-3410. [CrossRef] [PubMed]

16. Jia, C.; Lu, W.; Oyamada, J.; Kitamura, T.; Matsuda, K.; Irie, M.; Fujiwara, Y. Novel Pd(II)- and Pt(II)-catalyzed regio- and stereoselective trans-hydroarylation of alkynes by simple arenes. J. Am. Chem. Soc. 2000, 122, 7252-7263. [CrossRef]

17. Jia, C.; Pao, D.; Oyamada, J.; Lu, W.; Kitamura, T.; Fujiwara, Y. Efficient activation of aromatic C-H bonds for addition to C-C multiple bonds. Science 2000, 287, 1992-1995. [CrossRef] [PubMed]

18. Pastine, S.J.; Youn, S.W.; Sames, D. Pt ${ }^{\mathrm{IV}}$-catalyzed cyclization of arene-alkyne substrates via intramolecular electrophilic hydroarylation. Org. Lett. 2003, 5, 1055-1058. [CrossRef] [PubMed]

19. Nishizawa, M.; Takao, H.; Yadav, V.K.; Imagawa, H.; Sugihara, T. Mercuric triflate-(TMU) ${ }_{3}$-catalyzed cyclization of $\omega$-arylalkyne leading to dihydronaphthalenes. Org. Lett. 2003, 5, 4563-4565. [CrossRef] [PubMed]

20. Zhang, L.; Kozmin, S.A. Brønsted acid-promoted cyclizations of siloxyalkynes with arenes and alkenes. J. Am. Chem. Soc. 2004, 126, 10204-10205. [CrossRef] [PubMed]

21. Nevado, C.; Echavarren, A.M. Intramolecular hydroarylation of alkynes catalyzed by platinum or gold: Mechanism and endo selectivity. Chem.-Eur. J. 2005, 11, 3155-3164. [CrossRef] [PubMed]

22. Barluenga, J.; Trincado, M.; Marco-Arias, M.; Ballesteros, A.; Rubio, E.; González, J.M. Intramolecular iodoarylation reaction of alkynes: easy access toderivatives of benzofused heterocycles. Chem. Commun. 2005, 2008-2010. [CrossRef] [PubMed]

23. Gurtis, N.R.; Rassias, P.G.; Walker, A.J. A facile gold(I)-catalysed intramolecular alkyne hydroarylation approach to methyl 5-amino-2H-1-benzopyran-8-carboxylate derivatives. Tetrahedron Lett. 2008, 49, 6279. [CrossRef]

24. Qiu, W.-W.; Surendra, K.; Yin, L.; Corey, E.J. Selective formation of six-membered oxa- and carbocycles by the In(III)-activated ring closure of acetylenic substrates. Org. Lett. 2011, 13, 5893-5895. [CrossRef] [PubMed]

25. Wang, Y.; Ji, K.; Lan, S.; Zhang, L. Rapid access to chroman-3-ones through gold-catalyzed oxidation of propargyl aryl ethers. Angew. Chem. Int. Ed. 2012, 51, 1915-1918. [CrossRef] [PubMed]

26. Arcadi, A.; Blesi, F.; Cacchi, S.; Fabrizi, G.; Goggiamani, A.; Marinelli, F. Gold versus silver catalyzed intramolecular hydroarylation reactions of [(3-arylprop-2-ynyl)oxy]benzene derivatives. Org. Biomol. Chem. 2012, 10, 9700-9708. [CrossRef] [PubMed]

27. Eom, D.; Mo, J.; Lee, P.H.; Gao, Z.; Kim, S. Synthesis of vinyl sulfides and vinylamines through catalytic intramolecular hydroarylation in the presence of $\mathrm{FeCl}_{3}$ and AgOTf. Eur. J. Org. Chem. 2013, 533-540. [CrossRef]

28. Morán-Poladura, P.; Rubio, E.; González, J.M. Gold(I)-catalyzed hydroarylation reaction of aryl (3-iodoprop-2-yn-1-yl) ethers: synthesis of 3-iodo-2H-chromene derivatives. Beilstein J. Org. Chem. 2013, 9, 2120-2128. [CrossRef] [PubMed]

29. Eom, D.; Park, S.; Park, Y.; Lee, K.; Hong, G.; Lee, P.H. Brønsted acid catalyzed intramolecular hydroarylation for the synthesis of cycloalkenyl selenides and tellurides. Eur. J. Org. Chem. 2013, 2672-2682. [CrossRef]

30. Walkinshaw, A.J.; Xu, W.; Suero, M.G.; Gaunt, M.J. Copper-catalyzed carboarylation of alkynes via vinyl cations. J. Am. Chem. Soc. 2013, 135, 12532-12535. [CrossRef] [PubMed]

31. Murase, H.; Senda, K.; Senoo, M.; Hata, T.; Urabe, H. Rhodium-catalyzed intramolecular hydroarylation of 1-halo-1-alkynes: regioselective synthesis of semihydrogenated aromatic heterocycles. Chem. Eur. J. 2014, 20, 317-322. [CrossRef] [PubMed]

32. Xu, J.; Wang, Y.-L.; Gong, T.-J.; Xiao, B.; Fu, Y. Copper-catalyzed endo-type trifluoromethyl arylation of alkynes. Chem. Commun. 2014, 50, 12915-12918. [CrossRef] [PubMed]

33. Wang, Y.-L.; Zhang, W.-M.; Dai, J.-J.; Feng, Y.-S.; Xu, H.-J. Cu-catalyzed intramolecular hydroarylation of alkynes. RSC Adv. 2014, 4, 61706-61710. [CrossRef]

34. Fu, L.; Niggemann, M. Calcium-catalyzed carboarylation of alkynes. Chem. Eur. J. 2015, 21, 6367-6370. [CrossRef] [PubMed] 
35. Warner, A.J.; Lawson, J.R.; Fasano, V.; Ingleson, M.J. Formation of C( $\left.\mathrm{sp}^{2}\right)$-boronate esters by borylative cyclization of alkynes using $\mathrm{BCl}_{3}$. Angew. Chem. Int. Ed. 2015, 54, 11245-11249. [CrossRef] [PubMed]

36. Ding, D.; Mou, T.; Feng, M.; Jiang, X. Utility of ligand effect in homogenous gold catalysis: enabling regiodivergent $\pi$-bond-activated cyclization. J. Am. Chem. Soc. 2016, 138, 5218-5221. [CrossRef] [PubMed]

37. Lau, V.M.; Pfalzgraff, W.C.; Markland, T.E.; Kanan, M.W. Electrostatic control of regioselectivity in Au(I)-catalyzed hydroarylation. J. Am. Chem. Soc. 2017, 139, 4035-4041. [CrossRef] [PubMed]

38. Alonso-Marañón, L.; Martínez, M.M.; Sarandeses, L.A.; Pérez Sestelo, J. Indium-catalyzed intramolecular hydroarylation ofaryl propargyl ethers. Org. Biomol. Chem. 2015, 13, 379-387. [CrossRef] [PubMed]

39. Alonso-Marañón, L.; Sarandeses, L.A.; Martínez, M.M.; Pérez Sestelo, J. Sequential In-catalyzed intramolecular hydroarylation and Pd-catalyzed cross-coupling reactions using bromopropargyl aryl ethers and amines. Org. Chem. Front. 2017, 4, 500-505. [CrossRef]

40. Li, C.J.; Chan, T.H. Organic synthesis using indium-mediated and catalyzed reactions in aqueous media. Tetrahedron 1999, 55, 11149-11176. [CrossRef]

41. Shen, Z.-L.; Wang, S.-Y.; Chok, Y.-K.; Xu, Y.-H.; Loh, T.-P. Organoindium reagents: the preparation and application in organic synthesis. Chem. Rev. 2013, 113, 271-401. [CrossRef] [PubMed]

42. Zhao, K.; Shen, L.; Shen, Z.-L.; Loh, T.-P. Transition metal-catalyzed cross-coupling reactions using organoindium reagents. Chem. Soc. Rev. 2017, 46, 586-602. [CrossRef] [PubMed]

43. Onishi, Y.; Ito, T.; Yasuda, M.; Baba, A. Indium(III) chloride/chlorotrimethylsilane as a highly active Lewis acid catalyst system for the Sakurai-Hosomi reaction. Eur. J. Org. Chem. 2002, 1578-1581. [CrossRef]

44. Saito, T.; Nishimoto, Y.; Yasuda, M.; Baba, A. Direct coupling reaction between alcohols and silyl compounds: enhancement of Lewis acidity of $\mathrm{Me}_{3} \mathrm{SiBr}$ using $\mathrm{InCl}_{3}$. J. Org. Chem. 2006, 71, 8516-8522. [CrossRef] [PubMed]

45. Lee, P.H.; Lee, K.; Sung, S.-Y.; Chang, S. The catalytic Sakurai reaction. J. Org. Chem. 2001, 66, 8646-8649. [CrossRef] [PubMed]

46. Surendra, K.; Corey, E.J. Diiodoindium(III) cation, $\mathrm{InI}_{2}{ }^{+}$, a potent yneophile. Generation and application to cationic cyclization by selective $\mathcal{\omega}$-activation of C $\equiv$ C. J. Am. Chem. Soc. 2014, 136, 10918-10920. [CrossRef] [PubMed]

47. Liu, F.; Loh, T.-P. Highly stereoselective Prins cyclization of (Z)-and (E)- $\gamma$-brominated homoallylic alcohols to 2,4,5,6-tetrasubstituted tetrahydropyrans. Org. Lett. 2007, 9, 2063-2066. [CrossRef] [PubMed]

48. Hu, X.H.; Liu, F.; Loh, T.-P. Stereoelectronic versus steric tuning in the Prins cyclization Reaction: synthesis of 2,6-trans pyranyl motifs. Org. Lett. 2009, 11, 1741-1743. [CrossRef] [PubMed]

49. Shen, L.; Zhao, K.; Doitomi, K.; Ganguly, R.; Li, Y.-X.; Shen, Z.-L.; Hirao, H.; Loh, T.-P. Lewis acid-catalyzed selective [2 + 2]-cycloaddition and dearomatizing cascade reaction of aryl alkynes with acrylates. J. Am. Chem. Soc. 2017, 139, 13570-13578. [CrossRef] [PubMed]

50. Zhao, J.-F.; Loh, T.-P. Acid-catalyzed intramolecular [2 + 2] cycloaddition of ene-allenones: facile access to bicyclo[n.2.0] frameworks. Angew. Chem. Int. Ed. 2009, 48, 7232-7235. [CrossRef] [PubMed]

51. Li, B.; Lai, Y.-C.; Zhao, Y.J.; Wong, Y.-H.; Shen, Z.L.; Loh, T.-P. Synthesis of 3-oxaterpenoids and its application in the total synthesis of $( \pm)$-moluccanic acid methyl ester. Angew. Chem. Int. Ed. 2012, 51, 10619-10623. [CrossRef]

Sample Availability: Samples of the compounds 3aa-ja and 3ab-ae are available from the authors.

(C) 2018 by the authors. Licensee MDPI, Basel, Switzerland. This article is an open access article distributed under the terms and conditions of the Creative Commons Attribution (CC BY) license (http://creativecommons.org/licenses/by/4.0/). 